\title{
Phonon scattering and heat transfer by "diffusive" modes in solid $\mathrm{C}_{6} \mathrm{H}_{6}{ }^{*}$
}

\author{
O.I.Pursky ${ }^{\dagger 1}$, V.A.Konstantinov ${ }^{2}$ \\ 1 Taras Shevchenko National University of Kyiv, Faculty of Physics, 6 Glushkova Ave., Kyiv 03022, Ukraine \\ 2 Institute for Low Temperature Physics and Engineering of the National Academy of Science of Ukraine, \\ 47 Lenin Ave., Kharkov 61103, Ukraine
}

Received July 15, 2005, in final form November 19, 2005

\begin{abstract}
The isochoric thermal conductivity of solid $\mathrm{C}_{6} \mathrm{H}_{6}$ is described within a model in which the heat is transferred by phonons and above the phonon mobility edge by "diffusive" modes migrating randomly from site to site. The mobility edge $\omega_{0}$ is found from the condition, that the phonon mean-free path restricted by the examined mechanisms of scattering cannot become smaller than half the wavelength. The contributions of phononphonon, one and two-phonon scattering to the total thermal resistance of solid $\mathrm{C}_{6} \mathrm{H}_{6}$ are calculated under the assumption of additive contribution of different scattering mechanisms. Significant deviations from the dependence $\Lambda \propto 1 / T$ are explained by thermal conductivity approaching its lower limit.
\end{abstract}

Key words: molecular crystals, heat transfer, phonons, librons

PACS: $66.70+f, 63.20 . L s$

\section{Introduction}

At low temperatures, well below the Debye temperature of solids $\left(\Theta_{D}\right)$, heat transport in simple molecular crystals is adequately described by basic theoretical models [1]. However, in high temperature region $\left(T \geqslant \Theta_{\mathrm{D}}\right)$ experimental and theoretical understanding of thermal conductivity of molecular crystals remains incomplete. For example, at $T \geqslant \Theta_{\mathrm{D}}$, the classical theoretical models of heat transfer [1] predicted that thermal conductivity should be inversely proportional to temperature $(\Lambda \propto 1 / T)$, whereas experimental investigations of thermal conductivity of molecular crystals show considerable deviations from the above dependence [2]. Essentially all the basic concepts of heat transfer were created based on the studies of the simplest crystalline structure, i.e., atomic crystals. Therefore, the features typical of molecular crystals were not taken into account therein. One of the features that can affect the temperature dependence of thermal conductivity is the translation-rotation coupling. In the present study we wish to emphasize that thermal conductivity approaching its lower limit turns out to be another factor capable of determining the temperature dependence of thermal conductivity.

The purpose of this paper was to study the basic features of heat transfer in molecular crystals at $T \geqslant \Theta_{\mathrm{D}}$. Our previous measurements have revealed a considerable deviation of the isochoric thermal conductivity of solid $\mathrm{C}_{6} \mathrm{H}_{6}$ from the dependence 1/T [2]. The effect was explained qualitatively but we did not provide a quantitative interpretation. The present work continues investigating the observed effect on the solid $\mathrm{C}_{6} \mathrm{H}_{6}$. In the current study we analysed the temperature dependence of isochoric thermal conductivity of $\mathrm{C}_{6} \mathrm{H}_{6}$ using the model, which assumes that the heat is transferred by low-frequency phonons and above the mobility edge by "diffusive" modes, and taking into account phonon-phonon and phonon-rotation scattering.

*The paper submitted to the Proceedings of the conference "Statistical physics 2005: Modern problems and new applications" (August 28-30, 2005, Lviv, Ukraine).

†E-mail: pursky_o@ukr.net 


\section{The object}

Solid benzene under the pressure of its own saturated vapor has only one crystallographic modification: it has the orthorhombic spatial symmetry $P_{b c a}\left(D_{2 h}^{15}\right)$ with four molecules per unit cell $[3,4]$. Benzene melts at $278.5 \mathrm{~K}$ and the melting-caused change in the entropy is $\Delta S_{f} / R=4.22$ [5], which is much higher that Timmermans criterion for orientationally disordered phases. Here $R$ is universal gas constant. The high-temperature magnitude of the Debye temperature of $\mathrm{C}_{6} \mathrm{H}_{6}$ is $120 \mathrm{~K}[6]$. In the interval 90-120 K the second NMR moment of $\mathrm{C}_{6} \mathrm{H}_{6}$ drops considerably as a result of the molecule reorientations in the plane of the ring around the sixfold axis [7]. The activation energy of reorientational motion estimated from the spin-lattice relaxation time is $0.88 \mathrm{~kJ} / \mathrm{mole}$. The frequency of molecular reorientations at $85 \mathrm{~K}$ is $10^{4} \mathrm{~s}^{-1}$. On a further rise of the temperature it increases considerably, reaching $10^{11} \mathrm{~s}^{-1}$ near melting temperature. The basic frequency of the benzene molecule oscillations about the sixfold axis at $273 \mathrm{~K}$ is $1.05 \cdot 10^{12} \mathrm{~s}^{-1}$ [8].

At present, the thermal conductivity of solid $\mathrm{C}_{6} \mathrm{H}_{6}$ has been experimentally studied in the temperature range from $80 \mathrm{~K}$ to the melting temperature [2]. The isobaric thermal conductivity of solid $\mathrm{C}_{6} \mathrm{H}_{6}$ was also measured under the pressure above $100 \mathrm{MPa}[9]$.

\section{Model}

The calculation was performed based on the Debye's expression for thermal conductivity [1] using the approach of Roufosse and Klemens [10] who used the idea of a lower limit for the phonon mean free path:

$$
\Lambda=\frac{k_{\mathrm{B}}}{2 \pi^{2} v^{2}} \int_{0}^{\omega_{\mathrm{D}}} l(\omega) \omega^{2} \mathrm{~d} \omega,
$$

where $v$ is the polarization-averaged sound velocity, $\left(\omega_{\mathrm{D}}=\left(6 \pi^{2}\right)^{1 / 3} v / a\right)$ is the Debye frequency, $a^{3}$ is the volume per atom (molecule), and $l(\omega)$ is the phonon mean-free path.

Heat transfer in molecular crystals at $T \geqslant \Theta_{D}$ is determined mainly by phonon-phonon and phonon-rotational interactions. We assumed that the phonon-rotation relaxation time is determined by the one and two-phonon scattering processes [11]. Then, $l(\omega)$ - the combined phonon mean-free path determined by all of the examined mechanisms of scattering can be written as:

$$
l_{\Sigma}(\omega)=\left(\sum_{i} l_{i}(\omega)^{-1}\right)^{-1} .
$$

To explain the behavior of the thermal conductivity in solid $\mathrm{CH}_{4}$ and $\mathrm{CD}_{4}$, the authors of [11] used the analogy between molecular and spin systems. In a number of magnetic crystals the thermal conductivity was observed to increase above the magnetic phase transition. The reason for these anomalies is the scattering of phonons by critical fluctuations of the short-range magnetic order above the Neel point. In molecular crystals an increase of the isochoric thermal conductivity with increasing temperature is due to the weakening of phonon scattering by fluctuations of the shortrange orientational order. By analogy, using the equations for one and two-phonon relaxation times [12], the phonon mean free path of each of the examined scattering mechanisms can be expressed as:

$$
\begin{aligned}
l_{u}(\omega) & =v / A T \omega^{2}, \\
l_{I}(\omega) & =\rho v^{5} / B^{2} \Lambda_{\mathrm{rot}} T \omega^{2}, \\
l_{I I}(\omega) & =\pi \rho^{2} v^{8} / C^{2} k_{\mathrm{B}} C_{\mathrm{rot}} T^{2} \omega^{4}, \\
A & =\frac{18 \pi^{3}}{\sqrt{2}} \frac{k_{\mathrm{B}} \gamma^{2}}{m a^{2} \omega_{\mathrm{D}}^{3}},
\end{aligned}
$$

where the Grüneisen parameter $\gamma=-\left(\partial \ln \Theta_{\mathrm{D}} / \partial \ln V\right)_{T}, l_{u}(\omega)$ is the phonon mean-free path determined by $U$-processes, $l_{I}(\omega)$ and $l_{I I}(\omega)$ are the phonon mean-free paths for one and two-phonon 
scattering, respectively, $m$ is the average atomic (molecular) weight, $B$ and $C$ are the constants of non-central intermolecular interactions, $\Lambda_{\text {rot }}$ is thermal conductivity of the orientational subsystem, $\mathrm{C}_{\text {rot }}$ is rotational heat capacity per unit volume. In the first approximation: $B=C^{2}[11]$. The coefficient $B$ can be found from the pressure dependence of melting temperature:

$$
B=-\left(\frac{1}{\chi_{T}}\right) \frac{\partial\left(\ln T_{f}\right)}{\partial P},
$$

where $\chi_{T}$ is the isothermal compressibility, $T_{f}$ is temperature of the phase transition, $P$ is pressure. Thermal conductivity $\Lambda_{\text {rot }}$ can be found from the well-known gas-kinetic expression:

$$
\Lambda_{\mathrm{rot}}=\frac{1}{3} C_{\mathrm{rot}} a^{2} \tau^{-1}
$$

where $\tau$ is the characteristic time of orientational excitation transfer from one lattice site to another. This time was estimated from the relation $\tau \approx \hbar / \Delta E[13]$, where non-central part $\Delta E$ of the intermolecular interaction was calculated based on the expression given in [14] relating it to the phase transition temperature of $\mathrm{C}_{6} \mathrm{H}_{6}$.

By substituting (3)-(5) in (2), the combined phonon mean-free path can be expressed as follows:

$$
l_{\Sigma}(\omega)=\left(\frac{A T \omega^{2}}{v}+\frac{B^{2} \Lambda_{\mathrm{rot}} T \omega^{2}}{\rho v^{5}}+\frac{C^{2} k_{\mathrm{B}} C_{\mathrm{rot}} T^{2} \omega^{4}}{\pi \rho^{2} v^{8}}\right)^{-1} .
$$

Expression (9) is not applicable if $l(\omega)$ becomes of the order of or smaller than half the phonon wavelength: $\lambda / 2=\pi v / \omega$. A similar situation was considered previously for the case of $U$-processes alone [15]. Let us assume that in the general case:

$$
l(\omega)= \begin{cases}l_{\Sigma}(\omega), & 0 \leqslant \omega \leqslant \omega_{0} \\ \alpha \pi v / \omega=\alpha \lambda / 2, & \omega_{0}<\omega \leqslant \omega_{\mathrm{D}}\end{cases}
$$

where $\alpha$ is the numerical coefficient of the order of unity. The frequency $\omega_{0}$ can be found from the condition:

$$
\left(\frac{A T \omega_{0}^{2}}{v}+\frac{B^{2} \Lambda_{\mathrm{rot}} T \omega_{0}^{2}}{\rho v^{5}}+\frac{C^{2} k_{\mathrm{B}} C_{\mathrm{rot}} T^{2} \omega_{0}^{4}}{\pi \rho^{2} v^{8}}\right)^{-1}=\frac{\alpha \pi v}{\omega_{0}}
$$

It is equal to

$$
\omega_{0}=-\frac{u}{\left(-\eta+\sqrt{u^{3}+\eta^{2}}\right)^{1 / 3}}+\left(-\eta+\sqrt{u^{3}+\eta^{2}}\right)^{1 / 3}
$$

where the parameters $u$ and $\eta$ are:

$$
u=\frac{\pi \rho^{2} v^{7}}{3 C^{2} k_{\mathrm{B}} C_{\mathrm{rot}} T}\left(A+\frac{B^{2} \Lambda_{\mathrm{rot}}}{\rho v^{4}}\right) ; \quad \eta=-\frac{\rho^{2} v^{7}}{2 \alpha C^{2} k_{\mathrm{B}} C_{\mathrm{rot}} T^{2}} .
$$

The condition (11) is the well-known Ioffe-Regel criterion which implies localization. Therefore, we can assume that the excitations whose frequencies are above the phonon mobility edge $\omega_{0}$ are "localized" or "diffusive". Since completely localized modes do not contribute to the thermal conductivity, we supposed that the localization is weak and the excitations are capable of hopping from site to site diffusively, as was suggested by Cahill and Pohl [16].

If $\omega_{0}>\omega_{\mathrm{D}}$ the mean free path of all modes exceeds $\lambda / 2$ and the thermal conductivity is determined exceptionally by the processes of phonon scattering. At $\omega_{0} \leqslant \omega_{\mathrm{D}}$ the integral of thermal conductivity (1) is subdivided into two parts describing the contributions to the heat transfer from the low-frequency phonons and high-frequency "diffusive" modes:

$$
\Lambda=\Lambda_{\mathrm{ph}}+\Lambda_{\text {dif }} .
$$


In the high-temperature limit $\left(T \geqslant \Theta_{\mathrm{D}}\right)$ these contributions are:

$$
\begin{gathered}
\Lambda_{\mathrm{ph}}=\frac{k_{\mathrm{B}}}{2 \pi^{2} v^{2}} \int_{0}^{\omega_{0}} \frac{\omega \mathrm{d} \omega}{\frac{C^{2} k_{\mathrm{B}} C_{\mathrm{rot}} T^{2} \omega^{3}}{\pi \rho^{2} v^{8}}+\frac{A T \omega}{v}+\frac{B^{2} \Lambda_{\mathrm{rot}} T \omega}{\rho v^{5}}} \\
\Lambda_{\mathrm{dif}}=\frac{\alpha k_{\mathrm{B}}}{4 \pi v}\left(\omega_{\mathrm{D}}^{2}-\omega_{0}^{2}\right) .
\end{gathered}
$$

In the case of orientationally ordered phases equation (15) gives the well-known dependence $\Lambda \propto 1 / \mathrm{T}$ at $\omega_{0}>\omega_{\mathrm{D}}$

$$
\Lambda_{\mathrm{ph}}=\frac{k_{\mathrm{B}} \omega_{\mathrm{D}}}{2 \pi^{2} v A T}
$$

\section{Results and discussion}

As was mentioned above, at temperature close to or above the Debye temperature $T \geqslant \Theta_{\mathrm{D}}$ the thermal conductivity of pure crystals should be inversely proportional to temperature [1]. To obey the law $1 / \mathrm{T}$, the volume of the crystals should remain invariable, because the modes would otherwise change and so would the temperature dependence of the thermal conductivity $[9,17]$.

Nevertheless, isochoric studies of thermal conductivity of molecular crystals $[2,15,18]$ show a considerable deviation from this law. These discrepancies may be due to the thermal conductivity approaching its lower limit. The concept of the lower limit of thermal conductivity is based on the following: the mean free paths of the oscillatory modes participating in heat transfer are essentially limited but it cannot become smaller than half the phonon wavelength $\lambda / 2$, and the site-to-site heat transport proceeds as a diffusive process [10]. In this case the lower limit of the lattice thermal conductivity $\Lambda_{\min }$ can be written as [16]:

$$
\Lambda_{\min }=\left(\frac{\pi}{6}\right)^{\frac{1}{3}} k_{\mathrm{B}} n^{\frac{2}{3}} \sum_{i} v_{i}\left\{\left(\frac{T}{\Theta_{i}}\right)^{2} \int_{0}^{\Theta_{i} / T} \frac{x^{3} e^{x}}{\left(e^{x}-1\right)^{2}} \mathrm{~d} x\right\}
$$

The summation is over three (one longitudinal and two transverse) sound modes with the sound velocities $v_{i}$. $\Theta_{i}$ is the Debye cut-off frequency for each polarization in Kelvin's: $\left(\Theta_{i}=\right.$ $\left.v_{i}\left(\hbar / k_{\mathrm{B}}\right)\left(6 \pi^{2} n\right)^{1 / 3}\right), n=1 / a^{3}$ is the number of atoms per unit volume.

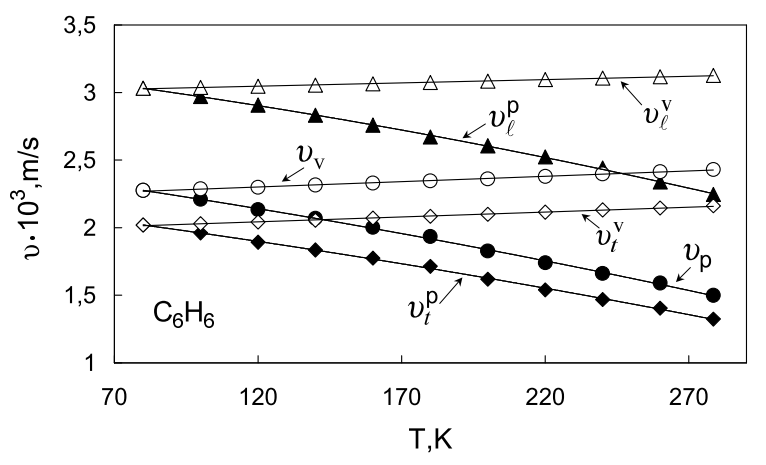

Figure 1. Calculated temperature dependence of the $\underset{P}{\text { isobaric and }} \underset{P}{\text { isochoric }}$ $\left(V_{\mathrm{mol}}=70.5 \mathrm{~cm}^{3} / \mathrm{mole}\right)$ sound velocities of solid $\mathrm{C}_{6} \mathrm{H}_{6} . v_{P}, v_{\ell}^{P}, v_{t}^{P}$ and $v_{V}, v_{\ell}^{V}, v_{t}^{V}$ are mean, longitudinal, and transversal phonon velocities for isobaric and isochoric conditions, respectively.

The calculated values of $\Lambda_{\text {min }}$ were as a rule considerably smaller than the experimental ones $[2,15,18]$. The most obvious reason for this difference is that the site to site transfer of the rotational energy was not taken into account. In molecular crystals the heat is transferred by mixed 
translation-rotation modes, whose heat capacity is saturated in proportion to the total molecular degree of freedom. Taking into account these features of molecular crystals, the lower limit of the thermal conductivity can be represented as [15]:

$$
\Lambda_{\min }^{*}=\frac{1}{2}\left(\frac{\pi}{6}\right)^{\frac{1}{3}}\left(1+\frac{z}{3}\right) k_{\mathrm{B}} n^{\frac{2}{3}}\left(v_{\ell}+2 v_{t}\right),
$$

where $v_{\ell}$ and $v_{t}$ are the longitudinal and transversal sound velocities, respectively, $z$ is the number of rotational degree of freedom.

To our knowledge, no experimental data are available on the sound velocity of solid $\mathrm{C}_{6} \mathrm{H}_{6}$. In this respect, it was calculated using the method described in [19]. The necessary data were taken from [20,21]. Figure 1 shows the calculated sound velocity of solid $\mathrm{C}_{6} \mathrm{H}_{6}$. The isochoric speed of sound corresponds to the molar volume $V_{\mathrm{mol}}=70.5 \mathrm{~cm}^{3} /$ mole.

The heat capacity of molecular crystal can be written as a sum of contributions from the translational $\mathrm{C}_{\mathrm{tr}}$ and rotational $\mathrm{C}_{\text {rot }}$ subsystems and a contribution due to the intramolecular vibrations of the atoms $\mathrm{C}_{\mathrm{in}}$ :

$$
C_{V}=C_{\mathrm{tr}}+C_{\mathrm{rot}}+C_{\mathrm{in}} .
$$

To separate the partial contributions to the heat capacity, we used the method described in [22]. The heat capacity at constant volume $C_{\mathrm{v}}$ is difficult to measure. In practice, it is recalculated, when data on thermal expansion $\beta$ [20] and Grüneisen parameter $\gamma[6]$ are available, from the values of the heat capacity at atmospheric pressure $C_{\mathrm{p}}[23]$ by using the known thermodynamic relation:

$$
C_{V}=\frac{C_{p}}{(1+\gamma \beta T)},
$$

$\mathrm{C}_{\mathrm{tr}}$ was calculated in the Debye approximation using the characteristic temperature $\Theta_{\mathrm{D}}=120 \mathrm{~K}$ obtained from the expression

$$
\Theta_{D}=v\left(\hbar / k_{\mathrm{B}}\right)\left(6 \pi^{2} n\right)^{\frac{1}{3}}
$$

and is close to $3 R$. $\mathrm{C}_{\text {in }}$ was calculated in the Einstein approximation using the intramolecular vibrational frequencies $\nu_{i}[6]$. $\mathrm{C}_{\text {rot }}$ was determined as $\mathrm{C}_{\mathrm{rot}}=\mathrm{C}_{\mathrm{v}}-\mathrm{C}_{\mathrm{tr}}-\mathrm{C}_{\mathrm{i} n}$. Our results for components of the heat capacity are shown in figure 2 . The heat capacity of librational subsystem $\mathrm{C}_{\text {rot }}$ constantly increases with the temperature increase, and at premelting temperatures approaching the values that are considerably larger than $3 / 2 R$, which is characteristic of a free three-dimensional rotator.

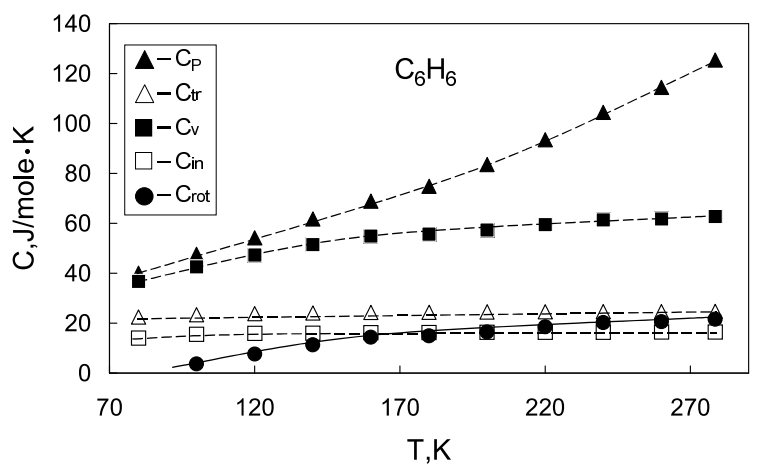

Figure 2. Temperature dependence of the heat capacity contributions in solid $\mathrm{C}_{6} \mathrm{H}_{6}$ : $\mathrm{C}_{\mathrm{p}}$ show the data [23] on the heat capacity at constant pressure, while $\mathrm{C}_{\mathrm{in}}, \mathrm{C}_{\mathrm{tr}}$, and $\mathrm{C}_{\mathrm{rot}}$ correspond to the calculated curves for the heat capacity at constant molar volume $V_{\text {mol }}=70.5 \mathrm{~cm}^{3} / \mathrm{mole}$ and for the intramolecular, translational, and rotational components of the heat capacity $\mathrm{C}_{\mathrm{v}}$, respectively.

To correctly compare the experimental results of thermal conductivity with the theory it is necessary to use data at constant density to exclude the effect of thermal expansion. The isochoric thermal conductivity of $\mathrm{C}_{6} \mathrm{H}_{6}\left(V_{\mathrm{mol}}=70.5 \mathrm{~cm}^{3} /\right.$ mole $)$ according to [2] is shown in figure 3 
(squares). The behavior of isochoric thermal conductivity $\Lambda_{\mathrm{v}}$ of solid benzene is inconsistent with the theoretical prediction. Satisfactory agreement with the classical law $1 / \mathrm{T}$ is observed only below $180 \mathrm{~K}\left(\Lambda_{\mathrm{v}} \sim \mathrm{T}^{-0.8}\right)$. At $T>180 \mathrm{~K}, \Lambda_{\mathrm{v}}$ is practically constant and even starts to grow slightly at premelting temperatures.

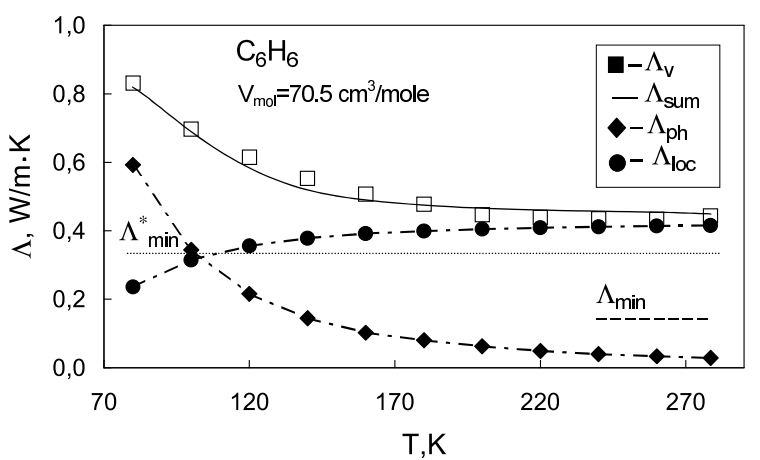

Figure 3. Isochoric thermal conductivity $\Lambda_{\mathrm{v}}$ of solid $\mathrm{C}_{6} \mathrm{H}_{6}$ (squares) [2]. The solid line is the fitting curve for isochoric thermal conductivity. $\Lambda_{\mathrm{ph}}$ and $\Lambda_{\text {dif }}$ are contributions of phonons and "diffusive" modes to heat transfer, respectively. The lower limits of the thermal conductivity $\Lambda_{\min }$ and $\Lambda_{\text {min }}^{*}$ calculated according to equations (18), (19).

The computer fitting of the thermal conductivity using equations (12)-(16) was performed using the least square method, varying the coefficients $A, B, C$, and $\alpha$. The parameters of the Debye model for thermal conductivity used in the fitting $(a, v)$, and the fitted values $A, B, C$, and $\alpha$ are listed in table 1.

Table 1. Parameters of the Debye model of thermal conductivity used in the fitting, and other quantities which were used for calculation.

\begin{tabular}{|c|c|c|c|c|c|c|c|}
\hline$V_{\mathrm{mol}}, \mathrm{cm}^{3} / \mathrm{mole}$ & $a, 10^{-8} \mathrm{~cm}$ & $v, \mathrm{~m} / \mathrm{s}$ & $\gamma$ & $\alpha$ & $A, 10^{-17} \mathrm{~s} / \mathrm{K}$ & $B$ & $C$ \\
\hline 70.5 & 4.9 & 2275 & 3.3 & 4.4 & 7.6 & 6.8 & 2.6 \\
\hline
\end{tabular}

The fitting results for isochoric thermal conductivity are shown in figure 3 (solid line). The same figure shows the contributions (dot-and-dash lines) to the heat transfer from the low-frequency phonons $\Lambda_{\mathrm{ph}}$ and the high-frequency "diffusive" modes $\Lambda_{\text {dif }}$ (calculated by equations (15), (16)). The dotted line shows the lower limit of thermal conductivity $\Lambda_{\text {min }}^{*}$ (19) calculated taking into account the possibility of site to site rotational energy transfer. The dashed line in the lower part of the figure is the lower limit of thermal conductivity $\Lambda_{\min }$ (18) calculated according to Cahill and Pohl within the framework of the Einstein model for the diffusive transfer of heat directly from atom to atom [16].

It is seen (figure 3) that the "diffusive" bahavior of oscillatory modes appears prior to $70 \mathrm{~K}$. As temperature rises, the amount of heat transferred by the "diffusive" modes increases, and at $110 \mathrm{~K}$ it becomes equal to the heat transferred by the low-frequency phonons. Above $150 \mathrm{~K}$ most of the heat is transported by the "diffusive" modes. As demonstrated in figure 3, our theoretical findings for thermal conductivity agree well with experimental data cited in [2]. The discussion of the lower limit of thermal conductivity of molecular crystals brings up inevitable question: should the siteto-site transport of the rotational energy of the molecules be taken into account? The minimum values of the experimental thermal conductivity $\Lambda_{\mathrm{v}}$ is 1.2 times higher than $\Lambda_{\text {min }}^{*}$ calculated by equation (19), and 2.5 times higher than $\Lambda_{\text {min }}$ calculated by equation (18). The above correlation between the $\Lambda_{\min }$ and $\Lambda_{\min }^{*}$ suggests a positive answer.

In high temperature region of existence of molecular crystals the contribution of librons $\Lambda_{\text {rot }}$ to the heat transfer is assumed to be relatively small $[2,15,24]$. The calculation of $\Lambda_{\text {rot }}$ according to (8) gives the values $1.42 \cdot 10^{-3} \mathrm{~W} / \mathrm{m} \cdot \mathrm{K}$ at $T=80 \mathrm{~K}$ and $1.5 \cdot 10^{-2} \mathrm{~W} / \mathrm{m} \cdot \mathrm{K}$ at $T=278 \mathrm{~K}$. These 
results prove the above assumption. At the same time, the role of librons in scattering processes is important $[2,24,25]$.

To answer questions as to basic peculiarities of phonon-rotational coupling in the solid $\mathrm{C}_{6} \mathrm{H}_{6}$, we have undertaken to separate the phonon-phonon and phonon-rotational contributions to the total thermal resistance. A number of molecular crystals have several solid phases, which substantially differ in the character of orientational ordering. If the non-central forces are strong and the temperature is low, there is a long-range orientational order in the location of molecular axes. The molecules perform small vibrations around the selected axes (librations), so that the motion of the neighbouring molecules is correlated and the collective orientation excitations (librons) propagate in the crystal. In the first approximation, the translation-orientation interaction in molecular crystals leads to an additional contribution to the thermal resistance $\mathrm{W}=1 / \Lambda[17]$.

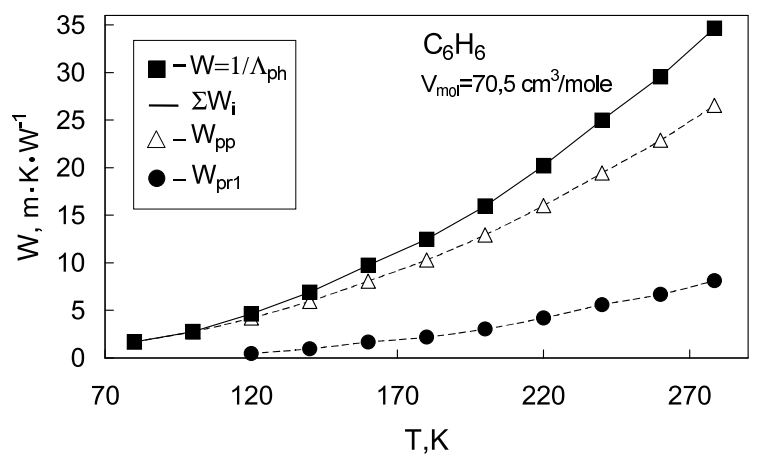

Figure 4. Contributions of the phonon-phonon scattering $W_{p p}$, and one-phonon $\mathrm{W}_{\mathrm{pr} 1}$ mechanisms of scattering to the total thermal resistance of solid $\mathrm{C}_{6} \mathrm{H}_{6}$. Square symbols indicate total thermal resistance $\mathrm{W}=1 / \Lambda_{\mathrm{ph}}$. The solid line shows the sum of thermal resistances $\mathrm{W}_{\mathrm{pp}}, \mathrm{W}_{\mathrm{pr} 1}$, and $\mathrm{W}_{\mathrm{pr} 2}$.

If the noncentral forces are relatively weak, and the temperature is high enough, the molecules can have a considerable orientational freedom. In this case a number of orientations are accessible to the molecule, which can pass from one orientation to another. In individual cases the limit of such reorientational motion can be a continuous rotation. The additional phonon scattering (compared to the phonon-phonon one) may originate in molecular crystals due to the collective rotational motion of molecules. As a rule, the unfreezing of molecular rotation is accompanied by an increase of the isochoric thermal conductivity due to the weakening of phonon-rotational scattering $[2,15]$. We assume that the contributions of different scattering mechanisms to the thermal resistance are additive [1]:

$$
\sum_{i} W_{i}=W_{p p}+W_{p r 1}+W_{p r 2}
$$

where $\mathrm{W}_{\mathrm{pp}}$ is the phonon-phonon thermal resistance, $\mathrm{W}_{\mathrm{pr} 1}$ and $\mathrm{W}_{\mathrm{pr} 2}$ are thermal resistances determined by one and two-phonon mechanisms of phonon-rotational scattering, respectively. Using (1) and (3)-(5) we have:

$$
\begin{aligned}
W_{p p} & =2 \pi^{2} v A T\left(k_{\mathrm{B}} \int_{0}^{\omega_{0}} \mathrm{~d} \omega\right)^{-1}, \\
W_{p r 1} & =2 \pi^{2} B^{2} \Lambda_{\mathrm{rot}} T\left(k_{\mathrm{B}} v^{3} \rho \int_{0}^{\omega_{0}} \mathrm{~d} \omega\right)^{-1}, \\
W_{p r 2} & =2 \pi C^{2} T^{2} C_{\mathrm{rot}}\left(v^{6} \rho^{2}\left|\int_{0}^{\omega_{0}} \frac{\mathrm{d} \omega}{\omega^{2}}\right|\right)^{-1} .
\end{aligned}
$$


Figure 4 shows the calculated results for thermal resistance $\mathrm{C}_{6} \mathrm{H}_{6}$. The total thermal resistance $\mathrm{W}=1 / \Lambda_{\mathrm{ph}}$ is shown with black squares. The solid curve is the sum $\sum_{i} W_{i}$ of the thermal resistances, calculated by equations $(23)-(25)$. The phonon-phonon component of the thermal resistance $W_{p p}$ increases with temperature. As temperature rises the thermal resistance $W_{p r 1}$ due to the onephonon scattering at rotational excitations of molecules also increases and becomes substantial after $120 \mathrm{~K}$. The two-phonon component $\mathrm{W}_{\mathrm{pr} 2}$ of the total thermal resistance is practically zero (it does not exceed $5 \cdot 10^{-5} \mathrm{~m} \cdot \mathrm{K} / \mathrm{W}$ ). From the aforesaid, it is clear that phonon-rotation scattering cannot be a reason for an increase of isochoric thermal conductivity of $\mathrm{C}_{6} \mathrm{H}_{6}$.

\section{Conclusions}

It is shown that the isochoric thermal conductivity of $\mathrm{C}_{6} \mathrm{H}_{6}$ can be described in a model, where the heat is transferred by phonons and above the phonon mobility edge by "diffusive" modes migrating randomly from site to site and taking into account the transfer of rotational energy between lattice sites. The total thermal resistance in molecular crystals is determined by phononphonon and phonon-rotation scattering mechanisms. In turn, the phonon-rotation relaxation time is determined by the one and two-phonon scattering. The contributions of phonon-phonon, one and two-phonon scattering to the total thermal resistance of solid $\mathrm{C}_{6} \mathrm{H}_{6}$ are calculated in supposition of additive contribution of different scattering mechanisms.

Based on these studies, it seems justified to conclude that the main reason for the essential deviations of the isochoric thermal conductivity of $\mathrm{C}_{6} \mathrm{H}_{6}$ from the dependence $1 / T$ is the additional heat transfer by "diffusive" modes. The latter is caused by the thermal conductivity approaching its lower limit.

\section{Acknowledgements}

This study was supported by the Ukrainian Ministry of Education and Science, Project No. 121-97.

\section{References}

1. Berman R. Thermal Conduction in Solids. Clarendon Press, Oxford, 1976.

2. Pursky O.I., Zholonko N.N., Konstantinov V.A., Fiz. Nizk. Temp., 29, 1021 (Low Temp. Phys., 2003, 29, 771).

3. Cox E.G., Cruickshank D.W.J., Smith J.A.S., Proc. R. Soc., 1958, 247, 1.

4. Bacon G.E., Curry N.A., Wilson S.A., Proc. R. Soc., 1964, 279, 98.

5. Ubbelohde A.R. Melting And Crystal Structure. Clarendon Press, Oxford, 1965.

6. Kitaigorodskii A.I. Molecular Crystals. Nauka, Moscow, 1971 (in Russian).

7. Andrew E.R., Eades R.G., Proc. Roy. Soc., 1953, A218, 537.

8. Parsonage N.G., Staveley L.A.K. Disorder in Crystals. Clarendon Press, Oxford, 1978.

9. Ross R.G., Andersson P., Bäckström G., Mol. Phys., 1979, 38, 377.

10. Roufosse M.C., Klemens P.G., J. Geophys. Res., 1994, 79, 703.

11. Krupskii I.N., Koloskova L.A., Manzhelii V.G., J. Low Temp. Phys., 1974, 14, 403.

12. Kawasaki K., Prog. Theor. Phys., 1963, 29, 801.

13. Mori H., Kawasaki K., Progr. Theor. Phys., 1962, 27, 529.

14. James H.M., Keenan T.A., J. Chem. Phys., 1959, 31, 12.

15. Konstantinov V.A., Fiz. Nizk. Temp., 2003, 29, 567 (Low Temp. Phys., 2003, 29, 422).

16. Cahill D.G., Watson S.K., Pohl R.O., Phys. Rev., 1992, B46, 6131.

17. Manzhelii V.G., Freiman Y.A. Physics of cryocrystals, Woodbury, AIP Press, New York, 1997.

18. Pursky O.I., Zholonko N.N., Konstantinov V.A., Fiz. Nizk. Temp., 2000, 26, 380 (Low Temp. Phys., 2000, 26, 278).

19. Pursky O.I., Zholonko N.N., Fiz. Tverd. Tela, 2004, 46, 1949 (Phys. Solid State, 2004, 46, 2015).

20. Pursky O.I., Zholonko N.N., Ukr. J. Phys., 2004, 49, 1105. 
21. Heseltine C.W., Elliot D.W., Wilson O.B., J. Chem. Phys., 1964, 40, 2584.

22. Isakina A.P., Prokhvatilov A.I., Fiz. Nizk. Temp., 1993, 19, 201 (Low Temp. Phys., 1993, 19, 142).

23. Kikoin I.K. Tables of Physical Data. Atomizdat, Moskow, 1976 (in Russian).

24. Konstantinov V.A., Manzhelii V.G., Smirnov S.A., Phys. St. Sol. (b), 1991, B163, 369.

25. Konstantinov V.A., Manzhelii V.G., Reviakin V.P., Smirnov S.A., Physica B, 1999 262, 421.

\title{
Фононне розсіяння та перенесення тепла “дифузними” модами в твердому $\mathrm{C}_{6} \mathrm{H}_{6}$
}

\author{
О.І.Пурський ${ }^{1}$, В.А.Константінов ${ }^{2}$ \\ 1 Національний університет ім. Т.Шевченка, фізичний факультет, вул. Глушкова 6, 03022 Київ, Україна
}

2 Фізико-технічний інститут низьких температур НАН України, 61103 Харків, пр. Леніна, 47

Отримано 15 липня 2005 р., в остаточному вигляді - 19 листопада 2005 р.

Ізохорна теплопровідність твердого $\mathrm{C}_{6} \mathrm{H}_{6}$ описується в рамках моделі, в якій тепло переноситься фононами, а вище від граничної рухливості - "дифузними" модами, що мігрують випадковим чином $з$ вузла на вузол. Границя фононної рухливості $\omega_{0}$ знаходиться із умови, що довжина вільного пробігу фононів, котра визначається розглянутими механізмами розсіяння фононів, не може стати меншою половини довжини хвилі. Внески фонон-фононного, одно та дво-фононного розсіяння в повний тепловий опір твердого $\mathrm{C}_{6} \mathrm{H}_{6}$ розраховано в припущенні адитивності внесків різних механізмів фононного розсіяння. Значні відхилення від залежності $\Lambda \propto 1 / T$ пояснюються наближенням теплопровідності до її нижньої границі.

Ключові слова: молекулярні кристали, перенос тепла, фонони, ліброни

PACS: $66.70+f, 63.20 . L s$ 
